\title{
Health Effects among the Family Caregivers of Cardiac Patients- A Case Study
}

\author{
${ }^{1}$ Ms.S.Rachel, ${ }^{2}$ Dr. A. Umesh Samuel Jebaseelan
}

\begin{abstract}
"Whenever someone takes care of someone, especially a loved one like me trying to help my son as much as possible, there is so much stress involved. Sometimes I have a really hard time trying to handle depression, stress plus my own medical problems. The stress and depression is unreal at times for me. I know it is for him also."- Voice of the caregiver.
\end{abstract}

\begin{abstract}
Family Caregivers provide care to their dear ones with a variety of controllable and uncontrollable conditions including advanced age, dementia, and heart problems. These experiences have a negative impact in their psychological, behavioral and physiological well being in their day to day life. Caregivers are often faced with continuous stressful events which may have direct consequences in their health and day to day life. Physicians and the interdisciplinary team who come across with such individuals have the responsibility to provide necessary care and intervention. In this report, the experience of a 56 year old woman who is the sole care giver for her son is explicated.The Son was diagnosed to have dilated cardiomyopathy, a condition in which the heart becomes weakened and enlarged. As a result, the heart cannot pump enough blood to the rest of the body and was advised to undergo heart transplantation. During his intense and unpredictable course, the caregiver's burden is complex and complicated by multiple competing priorities. This report illustrates care giver's health effects and the importance of identifying caregivers at risk of negative health outcomes and to intervene to alleviate the psychological distress associated with the care giving experience.
\end{abstract}

Key words: Caregivers, Cardiac patients and health effects

\section{Background of the Study}

Family care givers play a vital role in taking care of their dear ones and this case exemplifies the associated caregiver's psychological problems during cardiac treatment. Unlike professional caregivers such as physicians and nurses, informal caregivers, typically family members or friends, provide care to individuals with a variety of conditions, most commonly advanced age, dementia, and cardiac problems.Studies suggest that caregiving is deleterious to one's health, increased attention is being paid to the day to day well-being of caregivers. Compared to non-caregivers, caregivers often experience psychological, behavioral, and physiological effects that can contribute to impaired immune system function and coronary heart disease, and early death. Researchers examined heart risk factors in family members of cardiac patients and found that those who provided all or most of a patient's care had higher levels of risk factors for heart disease than non-caregivers and those who reported higher caregiver strain after six months were more likely to be depressed than those who provided less or no care. There is growing evidence that suggests stress and depression may play an important role in the development of cardiovascular disease. The encounter with caregiving affected all realms of caregivers' lives, and they experienced feelings of stress, vulnerability, and having to put their lives on hold; these feelings were often compounded by uncertainty. Kausar (2001) examined the relationship of the carer's primary appraisal (stress, threat, challenge and centrality), secondary appraisal (carers' perception of caregiving as control by self, control by others and uncontrollabity) and coping strategies in carers of physically disabled persons in Pakistan. Results indicated that the carer's primary appraisal of concerns and worries about physical disability had a strong relationship with anxiety and depression in carers. Financial concerns and carer-patient relationships were strong predictors of depression in carers. Rees J, Boyle CO, MacDonagh R . (2002)In analysing reports of 256 carers, it was found that caring for an incapacitated individual worsened health, impaired social and family life and increased stress, anxiety and depression among carers. Hop JW, Rinkel JE, Algra A, Gijn,JV (2006)in their study on quality of life in people affected by stroke and their partners, found the impact on the QOL of partners, prominently in the psychosocial domains. This is in keeping with increased emotional distress found in spouses or caregivers of people affected by stroke in general. Partners reported feelings of anxiety and uneasiness

Foster LW.et.al.,(2004)in their study on Survival of patients who have undergone allogeneic bone marrow transplantation: The relative importance of in-hospital lay care-partner support.found that Without a caregiver, transplant options may be limited and survival shortened. 


\subsection{Presenting signs and symptoms of Psychological distress}

Bland R.C Newman (1988).Kessler RC,Zhaos Melson C.B (1994) Offord Dr,Boyle M.H (1996) pointed that women are more prone than men to stress. While providing care to a loved one can be a rewarding experience, studies show that many family caregivers experience the same health problems. According to information culled by a non-profit called The Family Caregivers Alliance, caregivers are more prone to increased blood pressure and insulin levels than non-caregivers, and they may be at greater risk for heart attack. Also, caregivers suffer from clinical depression and insomnia at higher rates than the rest of the population ("Selected Caregiver Statistics" (San Francisco: Family Caregivers Alliance); available from http://www.caregiver.org/caregiver/jsp/content_node.jsp?nodeid=439; Internet; accessed July 2012). Grad and Sainsburg (1968) however found that the burden increased in proportion to the age and also the duration of the illness, but was not influenced by the sex of the patient. Financial problems were found to be present when the patient was a sole wage earner or when his illness prevented other family members from working. Burden was also encountered when social and leisure activities where interfered with.

Bakas T, et.al(2006) in their study on Family caregiving in heart failure have reported that Performing household tasks and managing patient behaviors were most difficult, and the caregiver's emotional and financial well-being, time for social activities, and general health had deteriorated.

Findings by Schulz and Beach. (1999), have recently shown that informal care giving is an independent risk factor for mortality. After adjusting for socio demographic factors, prevalent disease and subclinical cardiovascular disease, individuals who were providing care and experiencing caregiver strain had mortality risks that were higher than non caregiving controls (relative risk [RR], 1.63; 95\% confidence interval $[\mathrm{CI}], 1.00-2.65)$. These findings give research examining the role and impact of informal caregiving in all illness categories an added urgency.

Due to the heavy burden and responsibilities, carers of people affected by stroke experience depression and depression affects the Quality Of Life. Caregivers' psychological health is at heightened risk and social life becomes limited as well. Their physical health is also affected and satisfaction with the environment is also reduced. Patients' age also contributed to depression.

\section{Case Study}

Mr.X a 36 year old man diagnosed with dilated Cardiomyopathy was advised on heart transplantation .His wife having known that he had heart problem requiring continuous medication and care left him taking her two children.Mr.X was a state government employee who recently got his job permanent. The only care giver was his mother a widow aged 56 yrs.She reported extremely high levels of psychological distress,including anxiety,depression,frustration and extreme level of loneliness prior to the transplantation.

\subsection{Base line factors}

The patient was residing with his mother in sub urban part of Chennai. Therefore they were required to take a rented premise for his further follow up. Because the care giver was illiterate, unemployed and widow, she solely depended on her son's income for treatment and for their livelihood. Social support was limited to colleagues and relatives who rarely offered helping hand in terms of finance.

\subsection{Course of treatment}

The patient underwent heart transplantation successfully .the initial hospitalization and immediate recovery period was difficult and prolonged lasting approximately one month. After the initial hospital discharge, the patient was readmitted thrice having developed infections.

\section{Evaluating and managing health effects in Female Cardiac Caregivers}

Stressful events are unavoidable, but it is possible to assess the caregiver's psychological status when they visit the hospital with the patient. But generally, caregivers don't try to shift the focus on themselves as they care for the patients. In such a situation, as in this case, assessment should also be made focusing on the Caregiver. The caregiver in this case was assessed using Depression Anxiety Stress Scale 42 (DASS 42)Lovibond \& Lovibond (1995). Caregiver Burden Inventory (Caserta, Lund \& Wright, 1996). The World Health Organization Quality of Life (WHOQOL)-BREF (2004).

The DASS is a 42 item self-report inventory that yields 3 factors: Depression; Anxiety; and Stress. This measure proposes that physical anxiety (fear symptomatology) and mental stress (nervous tension and nervous energy) factor-out as two distinct domains. This screening and outcome measure reflects the past 7 days. Gamma coefficients that represent the loading of each scale on the overall factor (total score) are .71 for depression, .86 for anxiety, and .88 for stress. One would expect anxiety and stress to load higher than depression on the common factor as they are more highly correlated and, therefore, dominate the definition of this common factor (Lovibond and Lovibond, 1995). Reliability of the three scales is considered adequate and test-retest reliability is likewise considered adequate with .71 for depression, .79 for anxiety and .81 for stress 
(Brown et al., 1997). Exploratory and confirmatory factor analyses have sustained the proposition of the three factors ( $\mathrm{p}<.05$; Brown et al., 1997). The DASS anxiety scale correlates .81 with the Beck Anxiety Inventory (BAI), and the DASS Depression scale correlates .74 with the Beck Depression Scale (BDI).

24-item multi-dimensional questionnaire measuring caregiver burden with 5 subscales: (a) Time Dependence; (b) Developmental; (c) Behaviour; (d) Physical Burden; (e) Social Burden; (f) Emotional Burden. Scores for each item are evaluated using a 5-point Likert scale ranging from 0 (not at all disruptive) to 4 (very disruptive). Internal consistency reliability of each factor $0.85,0.85,0.86,0.73$, and 0.77 , respectively.

Facets incorporated within domains

1. Physical health Activities of daily Domain living, Dependence on medicinal substances and medical aids,Energy and fatigue, Mobility, Pain and discomfort, Sleep and rest, Work Capacity

2. Psychological Bodily image and appearance, Negative feelings, Positive feelings, Self-esteem, Spirituality / Religion / Personal beliefs, Thinking, learning, memory and concentration

3. Social relationships Personal relationships, Social support, Sexual activity

4. Environment Financial resources, Freedom, physical safety and security, Health and social care: accessibility and quality, Home environment, Opportunities for acquiring new information and skills, Participation in and opportunities for recreation / leisure activities, Physical environment (pollution / noise / traffic / climate), Transport Reliability of the WHOQOL-BREF was assessed using Cronbach's alpha. For the overall scale, alpha was 0.89 , and ranged from 0.74 to 0.77 for the individual domains. All values were above 0.70 , which demonstrated adequate internal consistency. Criterion relatedvalidity was assessed by correlating item and domain scores with the score of each of the two global items (Item 1: 'How would you rate your quality of life?' and Item 2: 'How satisfied are you with your health?'). All 24 remaining items were significantly correlated with Items 1 and 2 ( $p<0.01$ ), except for Item 4 , which was significantly correlated with Item $2(\mathrm{p}<0.01$ ), but not with Item 1. All domain scores were significantly $(\mathrm{p}<0.01)$ correlated with Item 1 (Pearson's $r$ ranged from 0.38 to 0.63 ), as well as Item 2 (0.32 to 0.47 ).

As a part of Intervention process, the caregiver's psycho social status was assessed using DASS,care giver burden scale and WHO bref Quality of life scale. In the linkert 5 point scale she scored 36, anxiety score was 42 and stress score was 42 which inferred extremely severe level of stress, anxiety and depression. In the care giver burden scale, she scored 56 which reveal extremely burn out situation of burden. To assess her quality of life scale, considering the Physical health Activities of daily living, she scored 9 having low physical health, considering Psychological Bodily image and appearance, she scored 8 revealing low psychological well being ,considering Social relationships Personal relationships, she scored 7 which infers that her social relationship has been affected and on Environment and Financial resources she scored 7 inferring that she is not satisfied with her environmental conditions.

\subsection{Challenges encountered by the Caregiver}

This caregiver had multiple care giving responsibilities, maintained two residences, to manage with limited finances and to look after her own health. Lacking the physical presence of a social support system, she shouldered the entire burden of her son's brief but intense outpatient care including: cleaning, coordinating medication and other treatment related activities. She struggled to maintain hope for the transplant's success, while living with the constant uncertainty of her son's survival, while she complained little, when asked; she reported severe emotional distress, significant fatigue, sleep impairment, and difficulty maintaining her focus and energy throughout the transplant trajectory.

\section{Intervention}

The mere assessment of the psycho social status of a care giver will not provide any positive changes in the life of the care giver.The assessment should be accompanied by a professional therapeutic intervention. Practitioner used medical crisis counseling as therapeutic intervention. It was a brief intervention the duration for each counseling session was about half an hour to one hour, which proceeded for a month were the client was told to address psychological and social problems which she encountered. Practitioner suggested various sources to apply for financial aid, which eased her from major burden. Practitioner counseled the client to use coping techniques in different stages to manage the stress of being a caregiver. Counseling helped the client to understand her reactions as normal response to stressful circumstance and to help her to function better.

\section{Case Study Follow up}

The patient expired during his fourth readmission, within a few months after his heart transplantation having complaints of various infections. The mother who was the caregiver had to go through a tremendous phase of stress in her life losing her only son, not knowing what to do losing everything in her life, she was totally depressed. She had to take up a job during her late 50's in a private concern to earn her daily living. 
Identifying the psychological, behavioral and physiological effects in a cardiac care giver will definitely pave the way for prevention of heart disease among the care giver. Care giver accompanies the patient in all their phase of life. Hence appropriate focus and awareness among the public for primary prevention and to emphasize a multi dimensional approach in research and practice should increase to attend these "hidden patients".

\section{Bibliography}

[1] Bakas T, et.al(2006) Family caregiving in heart failure retrieved from http://www.ncbi.nlm.nih.gov/pubmed/16708042 on September 2012

[2] Pinquart M., Sorensen S. (2003) Differences between caregivers and noncaregivers in psychological health and physical health: a meta-analysis. Psychol. Aging 2003;18:250-267.

[3] Schulz R., Beach S.R. (1999) Caregiving as a risk factor for mortality: the Caregiver Health Effects Study. JAMA 1999;282:22152219.

[4] Vitaliano P.P., Zhang J., Scanlan J.M.(2003) Is caregiving hazardous to ones physical health? A meta-analysis. Psychol. Bull. $2003 ; 129: 946-972$ 